Vietnam Journal of Mechanics, VAST, Vol.33, No. 3 (2011), pp. $194-202$

\title{
NEW CONCEPTS OF THEORY OF GEARING AND A METHOD FOR REVERSE SYNTHESIS OF THE GEARING
}

\author{
Nguyen Thien Phuc \\ Hanoi University of Science and Technology
}

\begin{abstract}
In this paper, the concepts of theory of gearing are developed by the synthesis of the results of our reseachs for years into theorical development and practical application of the generation of gear. A method of the reverse synthesis of the gearing is introduced in the third part.

Keywords: Theory of gearing, contact ellipse, elastohydrodynamic film, reverse synthesis.
\end{abstract}

\section{INTRODUCTION}

The contents of this paper cover the following main topics:

1) Gearing with the required contact ellipse

2) Gearing with the elastohydrodynamic film thickness

3) Generation of gear without the restriction of the use classic method of manufacturing tronics.

4) Innovation on the development of gear drives in the trend of the modern mecha-

Several new concepts of theory of gearing are based on the main results of our research into the mentioned topics [1] and on the lastest developments in its practical applications.

Follow these concepts we can develop new approaches for the design and manufacturing of the gears and propose a method for the reverse synthesis of the gearing.

\section{NEW CONCEPTS OF THE THEORY OF GEARING}

\subsection{Gearing with the required contact ellipse}

Up to now most people take great pains in research into the establishment of gearing with contact line. According to the viewpoint, the tooth surfaces are in the line contact at every instant. However, this is not practical because the inaccuracies in the manufacture and assembly are always occurred, so it is impossible to avoid. Such inaccuracies can cause stress concentrations on the edges and tips of the gear teeth.

There are many researchs into the localization of tooth contact. The tooth surfaces with the localized contact may be generated by shaping, hobbing or grinding etc. Among these we show concern for the approaches developed by the Gear Research Laboratory of 
the University of Illinois at Chicago $[2,3]$. However, all these approaches for the localization of teeth contact are passive solutions.

Applying the approach discussed in this paper we have determined the dimentions and orientation of the instantaneous contact ellipse and have controlled the solutions to increase its dimentions and to obtain a more favourable orientation. So that this is active solutions.

The developed theory of gearing with the required contact ellipse is based on the discussed approaches. According to this we considered 7 various cases for the generation [1] and proposed a method of the reverse synthesis of the gearing.

We may consider (see Fig. 1) four surfaces $\Sigma_{i}(i=1,2,3,4)$ and denote that the lines $t_{i}(i=1,2,3)$ are the lines of tangency between the surfaces $\Sigma_{1}$ and $\Sigma_{3}$, the surfaces $\Sigma_{2}$ and $\Sigma_{4}$ and the surfaces $\Sigma_{3}$ and $\Sigma_{4}$, respectively, at the common contact point M; $K_{i}(i=1,2,3)$ are the principal curvatures of two contacting surfaces $\Sigma_{1}$ and $\Sigma_{3}$, of two contacting surfaces $\Sigma_{2}$ and $\Sigma_{4}$, of two contacting surfaces $\Sigma_{3}$ and $\Sigma_{4}$, respectively, $\alpha_{i}(i=1,2,3)$ are angles formed by the line $t_{i}$ with the unit vector of the original direction.

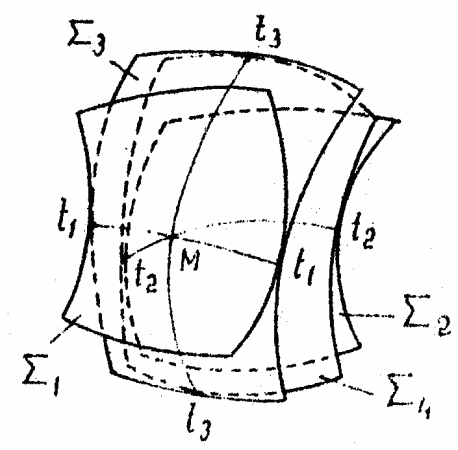

Fig. 1. Illustration of tangency of surfaces $\Omega_{I}(i=1,2,3,4)$

The determination of the instantaneous contact ellipse is based on the following equations [1]:

$$
\begin{gathered}
\operatorname{tg} 2 \beta_{*}=\frac{\sum_{i=1}^{3} K_{i} \sin 2 \alpha_{i}}{\sum_{i=1}^{3} K_{i} \cos 2 \alpha_{i}} \\
K_{*}=0,5 \sum_{i=1}^{3} K_{i} \pm 0,5\left[\sum_{i=1}^{3} K_{i}^{2}+2 \sum_{i=1}^{2} K_{i} K_{i+1} \cos 2\left(\alpha_{i+1}-\alpha_{i}\right)+2 K_{1} K_{3} \cos 2\left(\alpha_{3}-\alpha_{1}\right)\right]^{1 / 2}
\end{gathered}
$$

Here $\beta_{*}$ is the angle formed by the original direction with the principal direction of the equivalent curvature surface of two contacting surfaces $\Sigma_{1}$ and $\Sigma_{2} ; \mathrm{K}_{*}$ is the principal curvature of the equivalent curvature surface of two contacting surfaces $\Sigma_{1}$ and $\Sigma_{2}$. 
It is known, the equivalent curvature surface of two contacting surfaces shows the relationship betwee curvatures of two contacting surfaces.

By using equations (1) and (2) we may determine the principal curvatures and the principal directions of the equivalent curvature surface of two contacting surfaces $\Sigma_{1}$ and $\Sigma_{2}$. These principal directions have the same axes of the contact ellipse.

The derivation of the considerated cases for the generation of gear is based on the contact of four surfaces at the Fig. 1 . In total we considerated 7 cases and the $7^{\text {th }}$ case is presented as follow.

There are some approaches for the gearing with contact ellipse. This approach is based on the following considerations:

The surface $\Sigma_{1}$ and $\Sigma_{2}$ (see Fig. 1) are in line contact, but surface $\Sigma_{2}$ has been modified from $\Sigma_{3}$ to make it remained only one common contact point M. So we call unmodified surfaces $\Sigma_{3}$ "the theorical tooth surface" and modified surfaces $\Sigma_{3}$ "the practical tooth surface"

The approach discussed above allows reception of the approximate gearing with the contact ellipse. For this case, in [1] we obtained the following equations $(3-6)$, which are applied to determine the criteria for gearing quality:

$$
\begin{gathered}
V_{\sum}=\left[\left(2 K_{0}^{-1} B_{\tilde{p}}+V_{\tilde{p}}\right)^{2}+\left(2 K_{0}^{-1} B_{p}+V_{p}\right)^{2}\right]^{1 / 2} \\
V_{r 1}=\frac{1}{K_{0}}\left(B_{\tilde{p}}^{2}+B_{p}^{2}\right)^{1 / 2}, V_{r 2}=\frac{1}{K_{0}}\left[\left(B_{\tilde{p}}+K_{0} V_{\tilde{p}}\right)^{2}+\left(B_{p}+K_{0} V_{p}\right)^{2}\right]^{1 / 2} \\
t g \theta_{1 p}=\frac{B_{\tilde{p}}}{B_{p}}, \operatorname{tg} \theta_{2 p}=\frac{B_{\tilde{p}}+V_{\tilde{p}} K_{0}}{B_{p}+V_{p} K_{0}}, t g \theta_{p}=\frac{2 B_{\tilde{p}} K_{0}^{-1}+V_{\tilde{p}}}{2 B_{p} K_{0}^{-1}+V_{p}} \\
t_{21}^{\prime}=\frac{F_{\varphi}}{C}-\frac{1}{K_{0} C}\left(a_{1 \tilde{p}} B_{\tilde{p}}+a_{1 p} B_{p}\right) .
\end{gathered}
$$

where:

$$
\begin{aligned}
& B_{p}=K_{\tilde{p}} a_{2 p}-T_{p} a_{2 \tilde{p}}, B_{\tilde{p}}=K_{p} a_{2 \tilde{p}}-T_{p} a_{2 p}, \\
& a_{1 p}=V_{p} K_{p}+V_{\tilde{p}} T_{p}+a_{2 p}, a_{1 \tilde{p}}=V_{\tilde{p}} K_{\tilde{p}}+V_{p} T_{p}+a_{2 \tilde{p}}, \\
& F_{\varphi}=\left(\bar{\omega}_{e i} \times \bar{n}\right) \bar{V}-(\bar{\omega} \times \bar{n}) \bar{V}_{e i}, \\
& K_{p}=\sigma_{1 p}-\sigma_{2 p}, K_{\tilde{p}}=\sigma_{1 \tilde{p}}-\sigma_{2 \tilde{p}}, T_{p}=\tau_{1 p}-\tau_{2 p}, \\
& K_{0}=K_{p} K_{\tilde{p}}-T_{p}^{2}, C=i_{21}^{-1} \bar{V}_{e 2} . \bar{n}, \\
& a_{2 p}=V_{p} \sigma_{2 p}+V_{\tilde{p}} \tau_{2 p}+\omega_{\tilde{p}}, a_{2 \tilde{p}}=V_{\tilde{p}} \sigma_{2 \tilde{p}}+V_{p} \tau_{2 p}-\omega_{p}
\end{aligned}
$$

Here $V_{p}$ and $V_{\tilde{p}}$ are the projections of the linear relative velocity $\bar{V}$ on the axes $p$ and $\tilde{p}$, respectively, $\omega_{p}$ and $\omega_{\tilde{p}}$ are the projections of the angular relative velocity $\bar{\omega}$ on the axes $p$ and $\tilde{p}$, respectively, $\theta_{1 p}, \theta_{2 p}$, and $\theta_{p}$ are the angles that are formed by axis $p$ with the velocities $\bar{V}_{r 1}, \bar{V}_{r 2}$ of a contact point in its motion on the surfaces $\Sigma_{1}, \Sigma_{2}$ and the sum velocity $\bar{V}_{\Sigma} ; \mathrm{i}_{21}$ and i' 21 are the contact ratio and its differential; $\bar{V}_{e 1}$ and $\bar{V}_{e 2}$ are the linear velocities of the contact point in rotation about axes $O_{1}$ and $O_{2}$ respectively; $\tau_{i p}$ and $\sigma_{i p}, \sigma_{i \tilde{p}}$ are the geodetic twist of the line on the surface $\sum_{i}(i=1,2)$ and the normal curvatures of the surfaces $\sum_{i}(i=1,2)$ in the directions of axes $p$ and $\tilde{p}$, respectively. 


\subsection{Gearing with the elastohydrodynamic film thickness}

There is a contrary situation such as this usually occurred in the bearing. On the one hand, the area of the contact point between the surfaces is very small, but on the other hand, the capacity of the bearing is very high. What is the explanation of this situation?

The comprehensive description of this situation was given by academician P.L. Kapitsa [4]. He developed the theory of the elastohydrodynamic film with the round contact point.

In the paper [5] we expanded Kapitsa's theory into the theory of the elasto hydrodynamic film between the surfaces with ellipse contact.

Finally, we obtain the following formula for the dimensionless load paramter $W_{*}$.

$$
W_{*}=\frac{12 \sqrt{2}}{H_{*}^{1 / 2}}\left(\frac{\phi_{1}}{\varepsilon}\right)^{1 / 2} \sum_{n=1}^{3}(-1)^{n-1}(\alpha L)^{n-1} n^{-1} K_{n},
$$

where:

$$
\begin{aligned}
& K_{1}=1,466628 ; K_{2}=0,130712 ; K_{3}=0,024576 ; \\
& W_{*}=\frac{W}{\mu_{0} V_{\Sigma} R}, H_{*}=\frac{h_{n}}{R}, L=\frac{6 \sqrt{2} \mu_{0} V_{\sum} R^{1 / 2} \phi_{1}^{1 / 2}}{h_{n}^{3 / 2}}, \\
& \phi_{1}=\frac{\varepsilon \sin ^{2} \theta}{(2+3 \varepsilon)^{2}}+\frac{\cos ^{2} \theta}{(2 \varepsilon+3)^{2}} .
\end{aligned}
$$

Here $W$ is the load normal to surfaces, $N ; h_{n}$ is the minimun film thickness, cm; $\mu_{0}$ is the absolute viscosity at standard at mospheric pressure, $\mathrm{Ns} / \mathrm{cm}^{2} ; \alpha$ is the pressure - viscocity exponent, $\mathrm{cm}^{2} / \mathrm{N} ; \varepsilon \approx(a / b)^{-3 / 2}$.

In the paper [6] we have derived a formula for the dimensionless film thickness.

$$
H_{*}=2,36 V_{\Sigma_{*}}^{2 / 3} \phi_{1}^{1 / 3}
$$

where: $H_{*}=h_{n} / R, V_{\Sigma_{*}}=\mu_{0} \alpha V_{\Sigma} / R$.

The applied results of this theory allows new developments in the synthesis of the gearing, in the design, and in the manufacturing of the improved gear drives.

According to the obtained results in reseach the recommendations for the conditions of the axistence of a elastohydrodynamic film thickness are following:

- Select only a small difference between the curvatures of the concave and convex mating gear tooth surfaces.

- We have to emphasize that the orientation of contact ellipse is favourable. This orientation is determined by angle between the minor axis of the contact ellipse and the direction of the contact path. This angle is smaller as much as possible.

- The instantaneous contact ellipse can move with the sufficient speed.

We also carried out the experimmental research into the influence of the various parameters on the coefficient of friction between the surfaces with the contact ellipse [1].

Our investigation resulted in the following approximate equation.

$$
f=1,489 V^{-0,3} V_{\Sigma}^{-0,15} \nu^{-0,13} R^{-0,24}
$$


Here $V$ and $V_{\sum}$ are the sliding velocity and the total velocity, $\mathrm{cm} / \mathrm{s} ; R$ is the equivalent radius of curvature, $\mathrm{cm} ; \nu$ is lubricant absolute viscosity, $\mathrm{Ns} / \mathrm{cm}^{2}$.

\subsection{Generation of gear without the restriction of the use classic method of manufacturing}

Now, assume that the generated surface $\sum_{2}$ (the gear tooth surface) is represented by the family of lines of contact between the generating surface $\sum_{s}$ (the cutter tooth surface). The generating surface $\sum_{s}$ is represented by the family of shaper line of the cutter tool. This is the imaginary surface. It is difficult to manufacture this cutter tool exactly.

For that reason the previous designs of the tooth surfaces can be generated only by the simple kinds of the cutter tooth surfaces. So that we cannot widely use the various types in the making of the tooth profile so as to satisfy the growing requirements.

Nowadays, the information technology and the mechatronics have an effect on the modern manufacturing. There are some methods of the manufacture in the CNC machines, which are applied to generate the tooth gear profiles. Using this methods is free of the restriction mentioned above.

\subsection{Innovations in the development of gear drives in the trend of the modern mechatronics}

The modern systems of the numerical control machines and especially mobile equipment make several request for the gear drives. Among this the following are included:

- The backlash for a gear pair must be sufficient to permit free action under the most severe combimation of manufacturing tolerances and operating temperature variations. Howerve, the backlash is just the cause of a delay in the process of control. So that the process may be out of control in real time. Therefore, the annihilate of the mentioned backlash is the current task of the designer of the gear drives.

- The in crease of the gear mesh efficiency is the important factor, especially for the mobile and running by batteries equipment. Because it will decide how long is the continuous time for work and how about the loss of power transmittance. For the elastohydrodynamic film thickness between tooth surfaces, and the substitution of the sliding friction by the rolling friction at the contact surfaces.

- The gear drive's compact size is reseach goal of several studies of the gearing. Using the mentioned above measures we can strengthen the load capacity of the gear drive, consequently we can decrease its the size.

\section{A METHOD FOR REVERSE SYNTHESIS OF THE GEARING}

\subsection{Algorithms for solution to the reverse synthesis of the gearing}

Assuming that the surface $\Sigma_{1}$ is known and we desire the position, the size and the moving direction of the contact ellipse are available on request. So the task is that we need to find a surface that is conjugated to the surface $\Sigma_{1}$ and is satisfied with the mentioned demands.

The following illustrative example is related to the $7^{\text {th }}$ case that was considerated above. For this case we may establish relations with the different curvatures of the couple 
two surfaces $\Sigma_{2}$ and $\Sigma_{3}[1]$.

$$
\begin{gathered}
k_{p}=0,5 \cos ^{-2} 2 \gamma_{p}\left[\left(K_{1 p}-K_{p}^{*}\right)\left(\cos 2 \gamma_{p}+\cos 4 \gamma_{p}\right)+2\left(K_{1 \tilde{p}}-K_{\tilde{p}}^{*}\right) \sin ^{2} \gamma_{p}-T_{1 p} \sin 4 \gamma_{p}\right] \\
k_{\tilde{p}}=0,5 \cos ^{-2} 2 \gamma_{p}\left[\left(K_{1 p}-K_{p}^{*}\right)\left(\cos 4 \gamma_{p}-\cos 2 \gamma_{p}\right)+2\left(K_{1 \tilde{p}}-K_{\tilde{p}}^{*}\right) \cos ^{2} \gamma_{p}-T_{1 p} \sin 4 \gamma_{p}\right] \\
t_{p}=0,5 \cos ^{-2} 2 \gamma_{p}\left[\left(K_{1 p}-K_{p}^{*}\right) \sin 2 \gamma_{p}-\left(K_{1 \tilde{p}}-K_{p}^{*}\right) \sin 2 \gamma_{p}+2 T_{1 p} \cos 2 \gamma_{p}\right]
\end{gathered}
$$

where

$$
\begin{aligned}
& K_{p}^{*}=K_{p} \cos ^{2} \gamma_{p}+K_{\tilde{p}} \sin ^{2} \gamma_{p}+T_{p} \sin 2 \gamma_{p}, \\
& K_{p}^{*}=K_{p} \sin 2 \gamma_{p}+K_{\tilde{p}} \cos ^{2} \gamma_{p}-T_{p} \sin 2 \gamma_{p}, \\
& K_{1 p}=\sigma_{1 p}-\sigma_{3 p}, K_{1 \tilde{p}}=\sigma_{1 \tilde{p}}-\sigma_{3 \tilde{p}}, T_{1 p}=\tau_{1 p}-\tau_{3 p}, \\
& \operatorname{tg} 2 \gamma_{p}=\frac{2 T_{p}}{K_{p}-K_{\tilde{p}}} .
\end{aligned}
$$

Here $\tau_{3 p}$ and $\sigma_{3 p}, \sigma_{3 \tilde{p}}$ are the geodetic twist of the line on the surface $\Sigma_{3}$ and the normal curvatures of the surface $\Sigma_{3}$ in the directions of axes $\mathrm{p}$ and $\tilde{p}$ respectively; $t_{p}$ and $k_{p}, k_{\tilde{p}}$ are the geodesic twist of the line on the equivalent curvature surface $\left(\Sigma_{2 / 3}\right)$ of two contacting surfaces $\Sigma_{2}$ and $\Sigma_{3}$ and the normal curvatures of this surface $\left(\Sigma_{2 / 3}\right)$ in the directions of axes $p$ and $\tilde{p}$ respectively; $K_{p}^{*}$ and $K_{\tilde{p}}^{*}$ are the normal curvatures of the equivalent curvature surface $\left(\Sigma_{2 / 3}\right)$ in the principal directions; $\gamma_{p}$ is the angle that is formed by the direction of the major axis of the contact ellipse with the unit vector of the original direction.

Using equation (10 - 12) we may determine the position and the orientation (that is defined by the angle $\gamma_{p}$ ) of the contact ellipse. The center of the contact ellipse coincides with the contact point and its size depends on the elastic deformation. It is considered, as known from the experimental data.

It is known, the contact trace on the tooth surface is the important criterion for the evaluation on the quality of the gearing. The contact trace is formed as a set instantancous contact ellipse, when it moves along the path of contact on the tooth surface. In order to increase the dimensions of the contact ellipse and obtain a more favorable orientation of his motion we may select a rational collection of the value $K_{i}$ and $\alpha_{i}$ in the equations (1) and (2).

\subsection{Analysis of the criteria for the mesh quality of the gear drives}

There are several criteria for the meshing quality, such as: contact trace; contact stresses, coefficient of friction gear mesh efficiency, elastohydrodynamic film thickness parameter, change in the gear ratio, etc...

The dimensions of the instantaneous contact ellipse have affected the contact stresses and the contact trace on the tooth surfaces. So that we can use the dimensions of the contact ellipse as the common criterion instead of the contact stresses and the contact trace.

The existence of the elastohydrodynamic film thickness is the important factor. Therefore, we can use the following dimensionless film parameter $\lambda$ as a criterion for the mesh quality of the gear

$$
\lambda=h_{n} /\left(S_{1}^{2}+S_{2}^{2}\right)^{1 / 2} .
$$


The film parameter is the ratio of the minimun film thickness to composite roughness, where $S_{1}$ and $S_{2}$ are the rms (root mean squate) roughness of the two surfaces in contact. The film parameter $\lambda$ can be use as an indicator of the existence of the elastohydrodynamic film between contacting surfaces. At $\lambda \geq 3$ minimal wear and the extremely long life can be expected.

In [1] we use the following formula for the gear mesh efficiency:

$$
\eta=1-f \frac{\bar{v} \cdot \bar{v}_{2}}{\bar{n} \cdot \bar{v}_{2}}
$$

where $\bar{v}$ is the unit vector of the sliding velocity; $\bar{v}_{2}$ is the unit vector of the relative velocity of the gear drive $2 ; \bar{n}$ is the unit common normal to the contact surfaces; $\mathrm{f}$ is the fruction coefficient.

Note that, because of the tooth surface is modified, the function of transmission errors is indeed a periodic parabolic function. Such a function results in the reduction of noise and vibrations. Therefore we need to obtain the level of maximal transmission gear ratio $i_{21}$ is substantially reduced and to assure that the value of its derivative $i_{21}^{\prime}$ always is minus and its absolute value is minimum. This is the conclusion of our study [7]. So that we can use the behaviour of as criteria of the mesh quality of the approximate gearing.

By analysing the mentioned above criteria we make a few remarks about the more important criterion. This is the existence of the elastohydrodynamic film thickness, because while there is this film the other criteria (such as the contact stresses, the friction coefficient, ect..) become a secondary matter.

\subsection{Illustrative examples} surface

* Generation of the approximate mesh gears by the modification of the contacting

The results of the mentioned $7^{\text {th }}$ case for the generation of gear can be applied to the resolution of a problem of a reduce unevenness of the gear ratio of the hypoid and bevel gears cutting by the face generating gear [8]. This result is found its application at the Moscow car factory "Lenin Komsomol"

* The development of a "roller gear"

A "roller gear" is new terminology. It used in the planetary cylcloid roller gear reducer. This is a new development of the mechanical products with the tendency to replace sliding priction by rolling fruction.

A sectional drawing of the planetary cycloid roller gear reducer is shown in Fig.2. There are four basic components: 1 - the put shaft with eccentric and bearing; 2 - cycloid disks; 3 - a ring gear housing with rollers; 4 - an output shaft with drive rollers.

The planetary cycloid pin gear reducer was first developed by Shanhikov [9]. Today, there are very few manufacturers producing the planetary cycloid pin gear reducer with some changes.

The special features of our proposals are following: In the paper [10] it is shown that the tooth profile of the disk (2) is not cycloid curve, but is the epitrochoid curve. The question is that, which sections of the epitrochoid curve to choose for the selection are the existence of the contact elastohydrodynamic film thickness between the contacting surfaces at every instant and the attendance of the continous and hamonious curve of the 


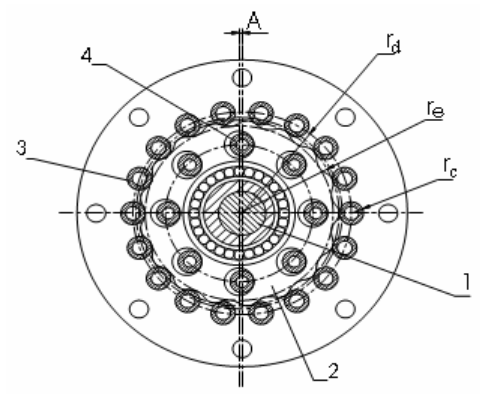

Fig. 2. The planetary roller gear reducer

profile of the disk for the silent movement, so that it is called "the harmonic disk". We can use the mathematical description of this curve for programming in order to manufacture the harmonic disk in the CNC machines.

The planetary roller gear reducers offer compact size, silent operation and heavy loads. These advantages are explained by follwing: The drives have a wide range of reduction rations; Due to the attendance of the roller the sliding friction usually present is replaced by rolling friction. Because of this lowered friction, these drives have efficiencis of $90 \%$ or higher; The existence of the convexo - concave contact, with a continuous contact hydrodynamic film of the conjugate gear profiles, plays the decisive role in attaining the goals; Due to their contruction the number of teeth under engagement is very high. So that these drives can carry higher loads; One factor that is considered in gear design for the modern mechanical power transmissions is backlask. In these resucers the backlask may be disposed of by using the two-disk or three-disk systems.

The applications of proposed roller gear in several domestic machine factories show the singificant advantages over conventional gears [11].

In comparison with two famous drives as Novikov helical gear in Russia and the harmonic drives in USA, we can understand that the planetary roller gear reducer is a successful combination of the advantages of two mentioned drives. Novikov proposed the patent for helical gearing of a circular-arc form. Novikov was awarded the Lenin Prize, but his concept had only limited application. The harmonic drives have a wide range of reduction ratios and many teeth in contact, but they have a weakness for the greatest difficulty in its manufacturing.

\section{CONCLUSIONS}

Base on the results of our research for years into the investigation of the theoretical and practical application of the gearing we have developed the following theories:

- Theory of gearing with the favourite contact ellipse.

- Theory of gearing with the elastohydrodynamic film thickness.

- Generation of gear without the restriction of the use classic method of manufacturing.

- Innovation in the development of gear drives in the trend of the modern mechatronics. 
Therefore we can determine the following orientations of the developed approaches for the design and manufacturing of the gears:

- Active generation of gear with the favourite orientaion and dimensions of the instantaneous contact ellipse.

- Consideration of the following criteria for the synthesis of the gearing:

+ Assume the tooth surfaces are convex and concave with a little difference in the curvatures.

+ Assume the angle between the moving direction of the instantancous contact ellipse and the minor axis of the contact ellipse is smallest.

- The reverse synthesis of the gearing is based on the satisfaction of user's requirements

- Search for the solutions of the substitution of the sliding friction by the rolling friction at the contact surfaces.

- The annihilate of the backlash for a gear pair is the current task of the designer of the gear drives for the control process without the delay.

That is the great significance from the point of view of the scientific and practical works.

\section{REFERENCES}

[1] Nguyen T.P., Foundations of theory of the elliptic contact of the space gear engagement, Doctor of Science thesis, Saint - Peterburg Technical University, (1978) (in Russian).

[2] Litvin F.L., Theory of Gearing, NASA RP - 1212, (1989).

[3] Litvin F.L., et al: Computerized Design an Generation of Low-Noise Helical Gears with Modified Surface Topology, Journal Mech. Des. ASME trans, 17(2) (1995).

[4] Kapitsa P.L., The theory of the hydrodynamic lubrication with rolling contact, Journal of the technical physic, 25(4) (1955) (in Russian).

[5] Dusev I.I., Nguyen T.P., Theory of the elastohydrodynamic lubrication with the elliptic contact, Surroundings and friction in the mechanisms, (3) (1977), (in Russian).

[6] Nguyen T.P., Elastohydrodynamic film thickness in the elliptic contact, Journal Science and Technology, Hanoi University of Technology, (1981) (in Vietnamese).

[7] Dusev I.I., Nguyen T.P., Optimal design for the approximate gear drives, Proceedings of the North Caucasian Scientific Centre of higher education - Science and Technique $N^{0} 4,(1977)$ (in Russian).

[8] Dusev I.I., Nguyen T.P., Determine the main quanlitative charatcristics of the hypoid and bevel gears cutting by the face generating gear, Proceedings of the North. Caucasian Scientific Centre of higher education - Science and Technique $N^{0} 2$, (1978) (in Russian).

[9] Koltrin N.I., Mechanics of machinery, Mashinostroenhie, Leningrad, (1972) (in Russian)

[10] Nguyen T.P., Planetary cycloid roller gear, Vietnam Journal of Mechanics, 24(2), (2002).

[11] Nguyen T.P., Nguyen T.D., Roller gear reducer, Proceedings of the th National Congress an Mechanics, (2002), (in Vietnamese). 